\title{
SIMULAÇÕES COMO APOIO A DECISÃO EM PROJETO PRODUTIVO PARA ASSENTAMENTO RURAL: UM ESTUDO DE CASO EM ROSÁRIO DO SUL - RS
}

\section{SIMULATIONS AS DECISION SUPPORT IN PRODUCTIVE PROJECT FOR RURAL SETTLEMENT: A CASE STUDY IN ROSARIO DO SUL - RS}

\author{
Alexandre Afonso Meyer* E-mail: alexandre.a.meyer@me.com \\ Mário Conill Gomes** E-mail: mconill@gmail.com \\ *Universidade Federal do Rio Grande do Sul (UFRGS), Porto Alegre, RS \\ ** Universidade Federal de Pelotas (UFPEL), Pelotas, RS
}

\begin{abstract}
Resumo: $O$ trabalho analisa riscos econômicos e financeiros para assentados rurais, através da combinação entre uma análise tradicional de negócios e uma análise simulada de resultados, visando promover o arbítrio de uma decisão melhor embasada. A pesquisa avaliou uma agroindústria, suprida por um sistema produtivo de leite em um assentamento rural, introduzindo elementos de aprofundamento a análise, na forma de simulações. O enfoque na produção e processamento de leite, como alternativa para um assentamento rural, foi projetado relacionando as variáveis de preços e custos das atividades. A construção do modelo simulado foi estruturada a partir do Valor Presente Líquido (VPL), submetido a simulações pelo método de Monte Carlo. As variáveis simuladas foram acolhidas por análise de sensibilidade, e os resultados obtidos no modelo demonstraram a probabilidade de $39,7 \%$ para um VPL negativo. Os elementos encontrados mostram que avaliações econômicas de sistemas simulados podem compor importantes ferramentas de apoio a projetos de investimentos de cunho coletivo em assentamentos rurais.
\end{abstract}

Palavras-Chave: Agroindústria. Leite. Monte Carlo. Risco.

\begin{abstract}
The paper analyzes economic and financial risks for rural settlers through the combination of a traditional business analysis and a simulated results analysis, aiming to promote the decision-making decision. The research evaluated an agribusiness, supplied by a milk production system in a rural settlement, introducing deepening elements to the analysis, in the form of simulations. The focus on milk production and processing, as an alternative to a rural settlement, was designed to relate price and activity cost variables. The construction of the simulated model was structured from the Net Present Value (NPV), submitted to simulations by the Monte Carlo method. The simulated variables were accepted by sensitivity analysis, and the results obtained in the model demonstrated the probability of $39.7 \%$ for a negative NPV. The evidence shows that economic evaluations of simulated systems can be important tools to support collective investment projects in rural settlements.
\end{abstract}

Keywords: Agribusiness. Milk. Monte Carlo. Risk.

\section{INTRODUÇÃO}

A reforma agrária como medida redutora de desigualdades sociais, contém uma sequência de ações que se estendem além da doação de terras para futuros produtores familiares. Uma série de elementos de estruturação formam os itens dos 
sistemas que resultam na viabilização de agricultores familiares, conforme comentam Batalha et al. (2005).

Renda suficiente e continuada, obtida da produção de terras destinadas `a reforma agrária, constitui um desafio de inúmeras faces e deve representar um dos elementos de importância dentro dos objetivos finais de qualquer política pública voltada ao apoio deste importante grupo de agricultores.

Construir bases capazes de fortalecer as iniciativas para o desenvolvimento econômico mais sustentável em assentamentos rurais no Brasil, representa um desafio diante do quadro mostrado nos principais trabalhos pesquisados (BERGAMASCO,1997; NORDER, 1997; LEITE, 2003; MONTE; PEREIRA, 2009; PICCIN, 2012) e compõem a justificativa do desenvolvimento deste estudo.

A abordagem do auxílio `a decisão na escolha de negócios, avançando para obtenção de maior chance de sucesso, através de um aprofundamento nas análises de operações projetadas, foi explorada no artigo como ponto central. E para isso, a pesquisa utilizou cenários simulados de negócios dentro de um assentamento rural. A contribuição do trabalho sugere ampliação na qualidade de parâmetros utilizados para avaliar atividades ou tecnologias projetadas em assentamentos rurais. E com isso, contribuir para a decisão pela escolha de determinada atividade ou tecnologia. O elemento fundamental para promover expansão de parâmetros para decisão, é a probabilidade de resultados negativos, encontrada por via de análise de risco via simulações. Os negócios projetados nas simulações; foram a produção e a industrialização de leite.

A hipótese levantada afirma que simulações podem promover uma aproximação para uma tomada de decisão com mais qualidade para estes produtores. Como ferramenta de intervenção utilizada para medir o risco e provocar um aprofundamento na análise tradicional, foi utilizada a técnica de Simulação de Monte Carlo. A técnica foi aplicada sobre o VPL resultante dos fluxos monetários projetados na produção e na industrialização de leite, por um período de dez anos. As variações no VPL, resultantes da aplicação das simulações, construíram as probabilidades de risco das atividades estudadas. A pesquisa foi conduzida utilizando como base, dois projetos técnicos estruturados, o de produção e o de industrialização de leite. Os projetos foram atrelados a um mesmo fluxo financeiro. E neste sentido, a pesquisa aponta para a formulação de um sistema que entrelaça duas atividades produtivas em 
um assentamento rural, expondo algumas relações de funcionamento da indústria instalada, integrada a um sistema produtivo de leite. Este conjunto foi examinado como uma alternativa factível ao grupo de assentados em questão. O objetivo central, foi debater se o risco do sistema simulado, pode compor uma base para qualidade na decisão de investimentos em assentamentos rurais. Portanto, avaliou-se a taxa de risco financeiro que o investimento enfrentaria, baseando-se nas premissas de operação elaboradas para o período de funcionamento.

Ao se combinar os resultados da análise tradicional por meio do VPL, com as simulações, o trabalho propõe a aplicação do recurso da modelagem Monte Carlo como uma ferramenta capaz de aprofundar o conhecimento a respeito do funcionamento de atividades produtivas projetadas e proporcionar melhor estruturação de decisões de negócios coletivos para pequenos produtores rurais.

\section{REVISÃO BIBLIOGRÁFICA}

\subsection{Aspectos das decisões administrativas na agricultura familiar}

Qualquer que seja o processo de tomada de decisão pelo homem, sempre estarão envolvidos dois tipos de elementos: os objetivos, e os elementos subjetivos próprios da natureza dos tomadores de decisão. $E$ este sistema não apresenta divisão, pois é constituído de um só corpo (BANA; COSTA, 1993, ANDRADE, 2000).

Para Simon (1997), existe na tomada de decisão, uma hierarquia de meios e fins, caracterizando não só o comportamento de organizações como o de indivíduos. Este comportamento de escolhas que seleciona preferências entre alternativas é chamado de racionalidade. Não obstante, esta corrente hierárquica de meios e fins, podem haver formulações incompletas, conflitos de interesse, contradições, e assim, a racionalidade do comportamento de organizações e dos indivíduos torna-se limitada ou incompleta, pois o comportamento racional envolve uma série de consequências que em uma ordem de preferência corresponde à alternativa escolhida.

Desta forma o autor descreve três etapas na tomada de decisão:

$1^{\circ}$ - Enumerar as alternativas.

$2^{0}$ - Avaliar as consequências destas estratégias.

3ํ- Comparar o conjunto de consequências de cada estratégia.

Revista Produção Online. Florianópolis, SC, v. 19, n. 4, p. 1120-1145, 2019 
Outros pontos são ainda relacionados com importância no comportamento para tomar decisões, como o tempo, o conhecimento, e o comportamento do grupo, que remete ao caso de cooperativas onde mesmo com a concordância de todo o grupo para determinado objetivo a ser alcançado, formas eficientes para buscar este objetivo requerem o conhecimento sobre as estratégias pessoais dos outros membros (SIMON, 1997).

Andrade (2000), comenta que os principais identificadores para uma tomada de decisão com qualidade dentro de um padrão perceptível de raciocínio são aqueles que geram garantia de realização de objetivos previamente estabelecidos através de meios reservados para tanto.

Payés e Silveira (1997), buscando o entendimento da racionalidade do produtor familiar, explicam que existem muitos tipos de agricultores e diferentes lógicas de raciocínio quanto ao uso de tecnologias, práticas produtivas e concluíram que o produtor familiar usa uma lógica capitalista na sua atividade com ressalva de assegurar o bem estar da família, reduzir o risco e manter a produção de autoconsumo, procurando inserção no mercado através de diferentes portfólios de produtos e uma relação diferenciada com a força de trabalho que representa sua família, procurando reduzir a penosidade do trabalho como uma forma de aumentar o conforto familiar.

A relação entre familiares, e a interdependência entre seus componentes conjuntamente com a atividade produtiva, faz com que as unidades familiares se organizem e funcionem como um sistema. E neste caso devem ser consideradas como um sistema aberto com relação ao meio ambiente, organizado para cumprir os objetivos e finalidades escolhidas pela família para a unidade produtiva (LIMA et al., 1995). Os elementos que formam este sistema são os insumos, os produtos e subprodutos, os serviços, os meios de produção que são os lotes, os animais, instalações, máquinas e equipamentos, e a força de trabalho. Estes fatores formam os fluxos de entradas e de saídas em uma unidade de produção. As ações administrativas do agricultor familiar consistem em gerir esses fluxos de maneira que o sistema seja mantido. $O$ processo de decisão do agricultor familiar implica em permanente cruzamento entre os objetivos do agricultor com sua família, e um conjunto de condicionantes bioclimáticos ligados à produção e à socioeconomia. 
Solano et al. (2001), ao avaliar os fatores que afetam a tomada de decisão em produtores de leite na Costa Rica, obteve em sua análise, que o nível de dedicação ‘a agricultura, o nível educacional, e o tamanho da propriedade, são características que influenciam fortemente para uma aproximação dos elementos que fundamentam as decisões. Desta maneira as decisões operacionais tendem a ser delegadas para algum membro da família, já as decisões técnicas são compartilhadas com um conselheiro técnico e com membros da família.

Abramovay (2012), resume três aspectos principais da racionalidade de produtores familiares: a) reter o máximo de lucro possível; b) minimizar os riscos; c) diminuir a penosidade do trabalho.

Fávero e Belfiore (2012), preconizam que a tomada de decisão se identifica diretamente com os objetivos da organização, de modo a minimizar as incertezas e os riscos inerentes ao processo, e ainda escolher a decisão mais eficaz. Para isso, a qualidade das informações é fundamental. A experiência prévia, a observação empírica e o consenso geral podem gerar boas decisões, mas o uso de ferramentas que forneçam resultados em forma de informações de qualidade para o processo de decisão, podem embasar fortemente este processo e suportar decisões com maior grau de certeza.

$\mathrm{Na}$ direção das ferramentas de análise para apoiar na composição dos elementos que estruturam a decisão dentro do caso estudado estão as avaliações feitas para projetos agropecuários e agroindustriais, especialmente os relacionados a assentamentos rurais. Portanto o prosseguimento da revisão bibliográfica trata alguns aspectos referentes as técnicas de avaliação de projetos.

\subsection{Avaliação econômica de projetos agropecuários}

Para Noronha (1987), Buarque (1991) e Nardelli e Macedo (2012), um projeto de investimento pode ser resumido a um fluxo de caixa abrangendo a vida útil do empreendimento em questão.

O fluxo de caixa como componente principal de um projeto representa o instrumento de relação da entrada e da saída de recursos financeiros em um determinado período. O prognóstico de excedentes ou de escassez de recursos pode 
ser construído em determinado momento através do fluxo de caixa de um projeto, e esta avaliação representa sua principal função (ZDANOWICZ, 1992).

Noronha (1987), Buarque (1991), Zdanowicz (1992) e Nardelli e Macedo (2012) concordam que o método tradicional de avaliação de projetos é o método chamado de Fluxo de Caixa Descontado. Onde se definem indicadores através de uma análise de Fluxo de Caixa, que consideram principalmente o valor do dinheiro no tempo, permitindo comparar quantias em instantes diferentes. Dentre estes indicadores, o Valor Presente Líquido (VPL), refere-se ao valor presente dos resultados dos fluxos de caixa futuros.

Em análise de viabilidade econômica na fruticultura, Ponciano et al. (2004), após construção do fluxo de caixa, utilizaram o índice VPL sob diferentes taxas mínimas de atratividade para mostrar a capacidade de diferentes culturas em superar condições de risco traduzidas sob a forma de Taxas Mínimas de Atratividade (TMA) crescentes. A avaliação partiu do resultado de VPL sob alto risco (TMA) para considerar a melhor opção para a região estudada. Avaliou-se neste trabalho dentro das opções de fruticultura, 11 cultivos submetidos a diferentes taxas mínimas de atratividade.

França, Holanda Junior e Souza Neto (2011), exploraram um modelo sustentável de atividades pecuárias no sentido de geração de renda em sistema silvipastoril. Utilizando na análise econômica do empreendimento produtivo o Valor Presente Líquido, os autores compararam os resultados de um sistema convencional da região com um sistema misto de exploração.

Moreira et al. (2007), examinaram em seu estudo de caso, a viabilidade econômica de agroindústria familiar rural, desde a sua estruturação inicial. A proposta de implantação de agroindústria de doces foi sustentada pela matéria prima de produção da agricultura familiar no município de São Miguel do Anta, na Zona da Mata Mineira. A primeira etapa do trabalho foi a construção do fluxo de caixa seguido dos cálculos de índices de resultado representados pelo VPL e pela Taxa Interna de Retorno (TIR). A taxa mínima de atratividade derivou da média ponderada das taxas de juros para financiamentos e da taxa de juros do capital próprio, obtendo $10 \%$ para TMA.

Tavares et al. (2011), projetaram um horizonte de três anos para o fluxo de caixa em três cultivos no agreste do estado de Pernambuco, e associaram a gestão Revista Produção Online. Florianópolis, SC, v. 19, n. 4, p. 1120-1145, 2019 
financeira aos recursos hídricos em atividades agrícolas, buscando a otimização destes recursos através da escolha que mostrou o maior valor, usando como indicador de resultado o VPL.

Lyra et al. (2010) trabalharam o tema do risco no cultivo de mamão em função do custo da irrigação, em experimentos no município de Linhares, no Espírito Santo. $\mathrm{O}$ autor construiu um fluxo de caixa e VPL para cada alternativa, utilizando duas variedades de cultivo sob cinco diferentes regimes hídricos.

As análises do VPL, permitem avaliar a viabilidade de um projeto em função dos resultados anuais de seu fluxo de caixa, da taxa mínima de atratividade, e do tempo de funcionamento do projeto. No entanto, uma análise de VPL não mostra outras possibilidades que não sejam aceitar ou recusar um projeto. Análises de possibilidades de resultados que vão além da aceitação ou recusa dos empreendimentos avaliados, são a justificativa da existência da teoria das opções reais (NARDELLI; MACEDO, 2011). Os autores afirmam que a principal incerteza em projetos agropecuários ocorre em função da flutuação dos preços futuros, e assim projetos com VPL positivo, em curto espaço de tempo podem gerar resultados negativos. Outro elemento de importância na consideração das incertezas para projetos agrícolas é o preço dos insumos específicos, seguidos de falhas na implantação de tecnologias e quebras de safra por eventos climáticos.

\subsection{Método de Simulação Monte Carlo}

Dentre as vantagens enumeradas por Chung (2004), na utilização de modelos simulados de análise de projetos estão, o pouco tempo gasto para experimentar situações diversas, o reduzido grau de exigência para realizar análise de modelos e a facilidade na demonstração destes modelos. Simulações não geram respostas fáceis a problemas complexos, portanto modelos por si mesmos não são capazes de resolver problemas.

Um projeto de simulação teve sucesso quando o modelo desenvolvido projetar uma representação adequada do sistema, e quando o estudo da simulação atingir os objetivos desejados pelos tomadores de decisão. O objetivo possível da Pesquisa operacional e de um de seus tópicos chamado de simulação é fornecer uma base racional e científica para apoiar tomadas de decisão dos administradores. Portanto, 0 
estudo da simulação deve fornecer ao tomador de decisão as respostas as suas dúvidas, e se ao iniciar uma simulação, esta não estiver alinhada com os objetivos dos tomadores de decisão o projeto estará fadado ao fracasso (CHWIF; MEDINA, 2010).

Gál et al. (2013), ao estruturar um modelo simulado para avaliar a gestão de propriedades produtoras de leite, afirmam que as simulações são apropriadas para otimizar os recursos sob um ambiente de estreitamento de margens, com aumentos nos custos de produção de leite.

A simulação do tipo Monte Carlo se enquadra na Teoria das Opções Reais para avaliação de projeto, teoria que é definida por Trigeorgis e Manson (1987), como um conjunto de opções reais. $E$ dentre estas opções, podem estar o cancelamento do investimento, o adiamento do investimento, ou de etapas, alterações de escala, e de custos, que não podem ser avaliados em análises tradicionais.

O conceito fundamental do método de Monte Carlo baseia-se na função de distribuição de probabilidades, e na função cumulativa de probabilidades de uma variável qualquer $(\boldsymbol{x})$. Ao se definir uma nova variável aleatória $(\boldsymbol{y})$ com distribuição uniforme sobre um intervalo fechado $(0,1)$, se estabelece uma relação entre as duas variáveis, sendo a variável $\boldsymbol{x}$, com distribuição aleatória própria e a variável $\boldsymbol{y}$, com distribuição uniforme entre 0 e 1 (ANDRADE, 2000).

O método de Monte Carlo se apoia matematicamente na teoria do Limite Central, pois baseia-se na capacidade de usar amostras para realizar inferências sobre parâmetros. As principais inferências que derivam da teoria do Limite central são segundo Stevenson (1981), as seguintes:

a) Se os dados sob amostragem têm distribuição normal, a distribuição das médias amostrais também será normal para todos os tamanhos de amostra.

b) Se os dados básicos têm uma distribuição que não é normal, a distribuição de médias amostrais será aproximadamente normal para grandes amostras.

Nas simulações de eventos discretos, a Simulação de Monte Carlo é considerada clássica. A técnica citada é capaz de recriar o funcionamento de um sistema real dentro de um modelo teórico (PRADO, 2009). Desta forma, define-se simulação de Monte Carlo como uma maneira de transformar um conjunto de números aleatórios em outro conjunto de números com a mesma distribuição da variável considerada.

Revista Produção Online. Florianópolis, SC, v. 19, n. 4, p. 1120-1145, 2019 
Bruni et al. (1998), ao examinarem projetos de investimento, tratam o método de Monte Carlo como uma alternativa ao problema de análise das variações que podem ocorrer nos índices econômicos como o VPL. Os autores comentam sobre a necessidade de haver cálculos formais com relação aos riscos de projetos que não são obtidos com o risco relacionado à média do VPL, ou seja, o risco expresso sob a forma do desvio padrão.

Silva (2004), projetou cenários utilizando simulações de Monte Carlo em uma agroindústria de laticínios de médio porte no estado de Minas Gerais. O objetivo da pesquisa foi examinar as decisões diante de diferentes cenários técnicos e de custo na fabricação de queijo. Os resultados mostraram fortes correlações dos preços de matéria prima com os custos de produção, e mostraram uma contribuição do método na utilização de cenários probabilísticos simulados, para auxilio nas decisões.

\section{METODOLOGIA}

O assentamento onde a pesquisa foi realizada, fica no município de Rosário do Sul/RS, onde no ano de 2000, foram fixadas sessenta famílias com a finalidade de reforma agrária. As famílias foram distribuídas em lotes contíguos de aproximadamente 3,66 hectares, totalizando 219,6 hectares; somando-se a área de 219,6 hectares, os assentados possuem uma área de aproximadamente 100 hectares composta por campo nativo, que foi destinada ao uso coletivo, e ainda uma área permanentemente inundada de aproximados 80 hectares. Assim, a área total do assentamento aproxima-se de 400 hectares. No ano de 2002, foi doada e instalada, no assentamento, uma indústria de laticínios, com capacidade para beneficiamento de leite em bebida láctea, queijo e leite pasteurizado.

A pesquisa foi construída a partir da realidade deste grupo de assentados. E a partir desta realidade, foram elaborados dois projetos complementares. $O$ primeiro projeto complementar, foi elaborado para prover a readequação das instalações da indústria de laticínios, com objetivo de colocar em operação a unidade de beneficiamento de leite. O segundo projeto trata do aproveitamento da área de uso coletivo dos assentados com a finalidade de produção de leite. Relacionando resultados técnicos e por consequência, resultados físicos e financeiros da produção de leite e do seu processamento na indústria. 
O modelo simulado, utilizou os resultados dos fluxos de caixa dos dois projetos para realizar uma análise combinada das variáveis de maior impacto, em um horizonte de dez anos. As variações do VPL simulado, são provenientes das variações nos valores das variáveis mais impactantes no VPL, segundo análise de sensibilidade realizada. Os valores das variáveis escolhidas, foram corrigidos, formando a base para geração das aleatorizações (simulações de valores). As variáveis foram simuladas pela técnica de Monte Carlo, utilizando para tanto, o VPL dos projetos que provém do fluxo de caixa projetado para as duas atividades (indústria de laticínios e produção de leite baseadas em projetos complementares elaborados pelos autores).

A modelagem considerou que o projeto industrial operaria recebendo cinco mil litros de leite integral ao dia, processados na forma de leite integral pasteurizado do tipo $\mathrm{C}$, embalados em sacos plásticos de um litro, queijo do tipo prato e bebida láctea embalada em pacotes plásticos de um litro.

A Taxa de juros utilizada no modelo de produção de leite, para fins de cálculo de despesas financeiras e financiamentos para produção, foi baseada na taxa de juros da linha Pronaf Mais Alimentos para a safra 2013/2014, que foi fixada em $2 \%$ ao ano. A Taxa Mínima de Atratividade (TMA) para cálculo do VPL na fase de produção foi obtida pela taxa média da poupança dos últimos 10 anos, que resultou em $5,7 \%$ ao ano (aa). A taxa de remuneração dos financiamentos, utilizada no modelo de industrialização de leite, baseou-se na taxa de juros da linha Pronaf Agroindústria, que para a safra $2013 / 2014$ foi fixada em $2 \%$ ao ano. A TMA utilizada foi $5,7 \%$ aa. Ambos os fluxos de caixa dos projetos, (produção e industrialização) foram unificados de maneira que a produção de leite com seu resultado de custos foi incorporada à indústria, gerando índices próprios que foram sintetizados em VPL como base de análise.

\subsection{Modelo Conceitual do Sistema Simulado}

Os cruzamentos das variáveis utilizadas na análise concorrem para diferentes resultados quando combinados, tomando em conta a produção e a industrialização do leite para a geração de um resultado final.

A representação conceitual do modelo como um todo (produção e industrialização) ilustra um sistema linear, estático e instável devido às mudanças que 
acontecem durante a evolução do rebanho (Tabela 1), na produção de leite. As variações devido a dinâmica do rebanho geram mudanças discretas tanto no resultado físico e monetário da produção de leite como no resultado físico e monetário da indústria, e desta forma acontece um afastamento do estado inicial do sistema. $\mathrm{O}$ modelo conceitual (Figura 1) ilustra a estrutura analisada.

Figura 1 - Modelo conceitual do sistema simulado

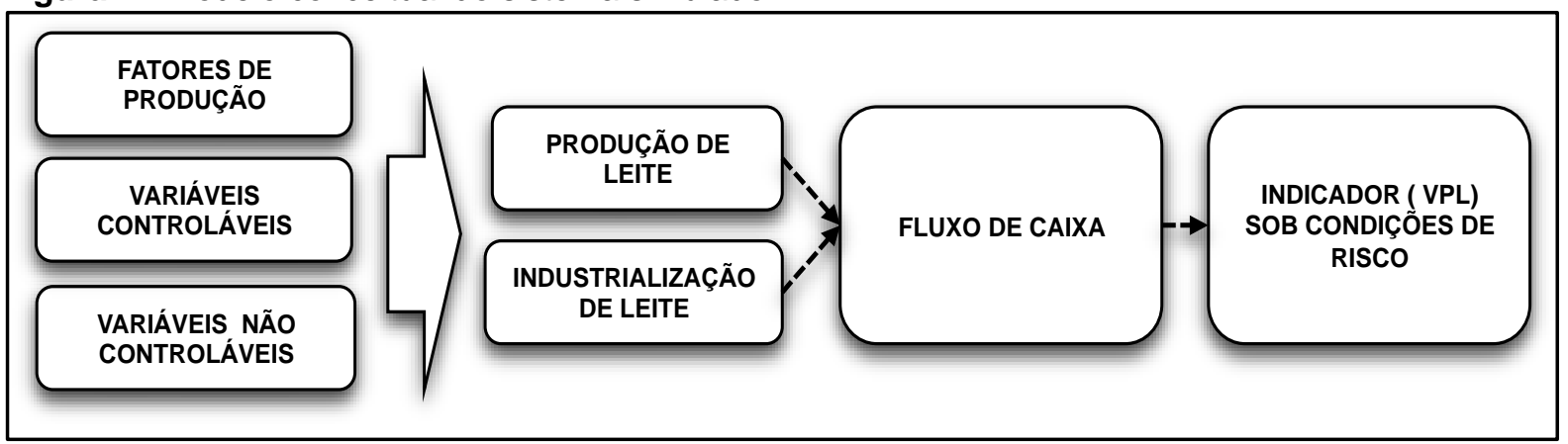

Fonte: Autores

\subsection{Modelo Analítico do Sistema Simulado}

Os resultados presentes de fluxo de caixa, foram construídos, utilizando preços atualizados, retirados dos projetos complementares.

O resultado presente do sistema, foi obtido pelo somatório dos resultados dos fluxos de caixa anuais de cada atividade. E assim o VPL foi calculado para cada ano durante os dez anos de avaliação na produção de leite e na sua industrialização.

O índice escolhido para representar o resultado de saída do modelo foi o VPL, por traduzir os resultados de caixa sinteticamente e por ser capaz de ser percebido facilmente pelos investidores na avaliação de uma tomada de decisão de investimento segundo Lapponi, (2000).

A fim de escolher as variáveis de entrada, utilizadas na simulação das duas atividades (produção e industrialização), procedeu-se uma análise de sensibilidade para identificar os elementos que mais impactam sobre o VPL do caixa unificado das atividades.

Dentro do modelo simulado, as variações no VPL são resultantes das variações dos elementos relacionados na análise de sensibilidade, pois as demais variáveis de produção foram elaboradas durante a construção dos projetos e permaneceram com seus valores anuais fixos, após cada rodada de simulação.

Revista Produção Online. Florianópolis, SC, v. 19, n. 4, p. 1120-1145, 2019 
Após a escolha das variáveis de entrada no modelo simulado, foram coletados dados históricos de preços destes elementos e foram identificados os outliers nos dados coletados através da análise do coeficiente de variação destes dados. As séries de preços das variáveis foram deflacionadas para a data de dezembro de 2013 utilizando como índice base para o deflacionamento o IGPM (Índice Geral de Preços de Mercado) coletado na FGV (Fundação Getúlio Vargas, 2014). Ainda nos procedimentos que antecederam a modelagem, as variáveis de entrada do modelo simulado, foram distribuídas nas suas frequências simples e acumulada, dentro de suas devidas classes. Na inferência para determinar o número de classes de cada distribuição foi utilizada a Regra de Sturges.

A geração de números aleatórios em distribuição normal, foi executada no programa estatístico StatPlus ${ }^{\circledR}(2009)$ e baseou-se na média aritmética das classes das variáveis escolhidas, e no desvio padrão das médias das classes de preços históricos corrigidos, em um intervalo de confiança de $99 \%$. Foram formulados dez mil registros de cada variável nos termos descritos acima. Portanto foram executadas dez mil rodadas de simulações no modelo.

O passo seguinte foi vincular em planilha eletrônica do programa Microsoft Excel® (2011), à distribuição acumulada de cada classe de valor da variável para obter a distribuição de probabilidade obtida através da combinação do número aleatório gerado, com a frequência de valores acumulados em cada classe de valor. O percentual de probabilidade do VPL resultante de dez anos de atividade, foi obtido em planilha eletrônica do $\operatorname{Exce|} \circledast$ (2011), pela inserção da função (2.3) a seguir:

$$
V P L<0=\operatorname{DIST} . \operatorname{NORM}(x ; \text { média; desv_padrão;cumulativo })
$$

A equação para a função de densidade de probabilidade em distribuição normal utilizada no Excel® está descrita abaixo (2.4).

$$
f(x, \mu, \sigma)=\frac{1}{\sqrt{2 \pi \sigma}} e^{\left(\frac{(x-\mu)^{2}}{2 \sigma^{2}}\right)}
$$

Cury (2004), afirma que sob a suposição de que uma distribuição de VPL seja aproximadamente normal, podem ser aplicadas as propriedades do cálculo da área 
sob a curva e sua probabilidade. Desta maneira foram criados os índices relacionados ao desvio padrão das classes para avaliar a distribuição provável de qualquer valor gerado dentro do intervalo encontrado.

Para descartar a presença de interação entre pares de variáveis de entrada, distribuídas sob a condição aleatória, foi efetuado um teste de correlação dentre os valores simulados.

Segundo Bussab e Morettin (2010), na averiguação do grau de dependência de variáveis quantitativas o coeficiente de correlação de Pearson $(\rho)$ é uma medida de associação mais adequada de relação linear.

As principais fontes utilizadas na coleta de preços históricos e dados técnicos utilizados para compor as simulações e para construir as variações nos preços, estão listadas a seguir: CEPEA - ESALQ; Instituto de Economia Agrícola - IEA - SP; Fundação Getúlio Vargas - FGV; ICEPA - SC; SEABI - PR; Atacado Leite - NUPEL, UEM-PR; Banco Central do Brasil - BACEN; Banco do Brasil - BB.

Os preços históricos coletados que serviram de base para projeções, representam o período que compreende os anos de 2003 a 2013.

A avaliação de risco proposta para os dois empreendimentos funcionando de forma contínua e utilizando o índice VPL dos fluxos de caixa, baseou-se nos trabalhos de Bruni et al. (1998) e Fernandes (2005).

\section{RESULTADOS E DISCUSSÃO}

A venda de produtos da industrialização de leite ficou distribuída da seguinte forma: a) Venda de leite, 1.000 .000 litros ao ano, b) Bebida láctea, 700.000 litros ao ano, c) Queijo, 35.000 quilos ao ano. As receitas foram obtidas pelos preços médios de venda dos produtos finais da indústria, multiplicados pelas quantidades projetadas de venda ao ano. Os preços projetados para executar a análise tradicional (VPL) foram: leite in natura, $R \$ 0,80$ por litro, leite industrializado tipo $C, R \$ 1,20$ por litro $e$ bebida láctea $R \$ 1,18$ por litro. O queijo tipo prato foi comercializado na projeção, a $\mathrm{R} \$ 21,16$ por quilo.

O valor negativo $(-11.979,00)$ do VPL referente a produção, indicou que o sistema produtivo de leite praticado no modelo projetado, combinando as premissas técnicas e orçamentárias utilizadas, não permitiram uma exploração eficiente do ponto 
de vista do Valor Presente. Ainda com relação ao VPL da produção de leite, deve se considerar que os juros praticados na TMA foram resultado da média aritmética, da taxa de rendimento da poupança, com base nos últimos 10 anos em pesquisa junto ao Banco do Brasil. A taxa média resultante foi de 5,7\% de rendimento ao ano.

Sob a avaliação econômica tradicional, a produção de leite no assentamento mostrou-se frágil nos moldes técnicos atribuídos e apontou para uma revisão nos elementos técnicos a fim de buscar mais eficiência produtiva e financeira a médio e longo prazos.

A fusão dos resultados de fluxo de caixa da produção e industrialização, foi executada através do somatório dos resultados líquidos do fluxo de caixa da produção, aos resultados líquidos do fluxo de caixa da industrialização, conforme mostrado na tabela abaixo (Tabela 1).

Se observou nos resultados da análise tradicional (VPL), que os preços médios obtidos e praticados na construção do fluxo de caixa, concederam viabilidade para as duas atividades, quando unidas.

Ao finalizar a fase de construção e análise tradicional dos projetos, foram executados os procedimentos referentes às simulações dos resultados econômicos. O primeiro passo para as simulações, foi a escolha das variáveis mais importantes. $O$ grau de impacto no VPL foi o critério usado. Assim, as escolhas das variáveis causadoras de maior mudança nos resultados econômicos foram escolhidas para a simulação. A análise de sensibilidade foi baseada na descrição de Bertolo (2009). As simulações consideraram as cinco variáveis mais importantes, conforme tabela abaixo (Tabela 2). 
Tabela 1 - Fluxo de caixa integrado (R\$ano)

\begin{tabular}{|c|c|c|c|c|c|c|c|c|c|c|}
\hline Ano & 1 & 2 & 3 & 4 & 5 & 6 & 7 & 8 & 9 & 10 \\
\hline Valor & $294.419,0$ & $281.340,0$ & $260.556,0$ & $275.811,0$ & $277.650,0$ & $313.454,0$ & $326.559,0$ & $337.820,0$ & $339.928,0$ & $462.872,0$ \\
\hline VPL & \multicolumn{3}{|c|}{$1.546 .272,4$} & & & & & & TMA & $5,70 \%$ \\
\hline
\end{tabular}

Fonte: autores 
Tabela 2 - Variação no VPL por produto avaliado

\begin{tabular}{lcc}
\hline \multicolumn{1}{c}{ Variável } & $\begin{array}{l}\text { Variação Relativa no VPL } \\
\text { para cada 1\% de Mudança no } \\
\text { Preço da Variável }\end{array}$ & $\begin{array}{l}\text { Variação Absoluta no VPL para } \\
\text { cada 1\% de Mudança no Preço da } \\
\text { Variável }\end{array}$ \\
\hline Leite IN & $6,3354 \%$ & $\mathrm{R} \$ 130.157,71$ \\
NATURA & $5,2576 \%$ & $\mathrm{R} \$ 108.015,59$ \\
Leite Tipo C & $3,6396 \%$ & $\mathrm{R} \$ 74.774,59$ \\
Bebida Láctea & $3,2390 \%$ & $\mathrm{R} \$ 66.544,79$ \\
Queijo & $0,7031 \%$ & $\mathrm{R} \$ 14.443,93$ \\
Concentrado & &
\end{tabular}

A análise de sensibilidade mostrou que variáveis exógenas (preços de venda dos produtos) provocaram maior impacto no resultado do VPL, o que deve elevar o risco do negócio, visto que são elementos que fogem ao controle dos produtores, pois, via de regra, produtores atuam como tomadores de preços, sujeitos a maioria das variações dos mercados.

As principais variáveis de impacto em sistemas agrícolas repousam sobre os preços dos produtos agropecuários e preços de insumos específicos (NARDELLI; MACEDO, 2011).

Mendes e Padilha Junior (2007), acentuam que uma característica fundamental dos preços dos produtos agropecuários é a instabilidade, afirmando que apresentam alto grau de variabilidade ao longo do tempo.

Neste caso, as chances de proteção contra grandes variações de preços, podem ser positivas na integração da produção com a indústria. No modelo simulado, a integração da produção com a industrialização, aponta para uma alternativa factível, pois, à medida que a produção cumpre o papel de abastecer a indústria, ela pode aumentar a estabilidade de custos de matéria prima. Minimizar grandes oscilações de mercado no preço do leite in natura, pode representar uma vantagem para qualquer integração.

A tabela abaixo, mostra o abastecimento de matéria prima para a indústria dentro do sistema modelado (Tabela 3).

O artigo foi elaborado dentro de um assentamento rural e o aproveitamento de mão de obra abundante dos produtores, reveste-se de uma importância social que justifica o empenho do estudo em unir uma estrutura previamente existente (indústria e a área de terra disponível), com os recursos de mão de obra de um assentamento rural. 
Tabela 3 - Produção de matéria prima (leite in natura)

\begin{tabular}{ccccccccccc}
\hline ANO & $\mathbf{1}$ & $\mathbf{2}$ & $\mathbf{3}$ & $\mathbf{4}$ & $\mathbf{5}$ & $\mathbf{6}$ & $\mathbf{7}$ & $\mathbf{8}$ & $\mathbf{9}$ & $\mathbf{1 0}$ \\
\hline $\begin{array}{l}\text { Suprimento da } \\
\begin{array}{l}\text { Industria pelo } \\
\text { assentamento }\end{array}\end{array}$ & $11 \%$ & $11 \%$ & $11 \%$ & $15 \%$ & $18 \%$ & $23 \%$ & $26 \%$ & $28 \%$ & $30 \%$ & $32 \%$ \\
\hline
\end{tabular}

Fonte: Autores

Na sequência da elaboração das simulações, foram construídos os testes nas séries de preços das variáveis escolhidas, para verificação de sua distribuição. Os coeficientes de variação $(C V)$ foram calculados para certificação de uma distribuição normal para os valores de preços históricos coletados. Para tanto, Chwif e Medina (2010) recomendam valores de coeficientes de variação abaixo do valor um (1). Os coeficientes encontrados nos dados de preços das variáveis estudadas foram: $C V=$ 0,3690 para o preço do leite ao produtor. $C V=0,9369$ para o preço do leite tipo $C$ no atacado. $C V=0,2199$ para o preço da bebida láctea. $C V=0,7652$ para preço do queijo. $C V=0,5291$ para preço do concentrado.

Examinados os coeficientes de variação, os preços médios utilizados na avaliação tradicional foram acrescidos de seu desvio padrão conforme tabela abaixo (Tabela 4), prosseguindo com a criação dos conjuntos de números aleatórios.

Tabela 4 - Preços médios e desvio padrão

\begin{tabular}{lcc}
\hline VARIÁVEL & MÉDIA DE PREÇO & DESVIO PADRÃO \\
\hline Concentrado & 0,7576 & 0,2128 \\
Leite in natura & 0,8050 & 0,2017 \\
Leite Tipo C & 1,2025 & 0,4839 \\
Bebida Láctea & 1,1892 & 0,1650 \\
Queijo & 21,1663 & 1,9967 \\
\hline
\end{tabular}

Fonte: Autores

A resposta ao risco financeiro das atividades unidas, foi elaborada a partir das simulações desenvolvidas em torno das variáveis de preços, que causaram maior impacto nos resultados (VPL). Os valores aleatórios foram criados através das médias e desvios padrão, provenientes dos preços históricos corrigidos, das variáveis escolhidas na análise de sensibilidade. Após a combinação dos valores referentes ao fluxo de caixa das atividades unidas, um VPL foi determinado para cada rodada no modelo de simulação, permitindo uma visão geral da distribuição do evento de saída do modelo (VPL), conforme demonstrado na figura abaixo (Figura 2). 
Figura 2 - Frequências do VPL em 10.000 rodadas simuladas

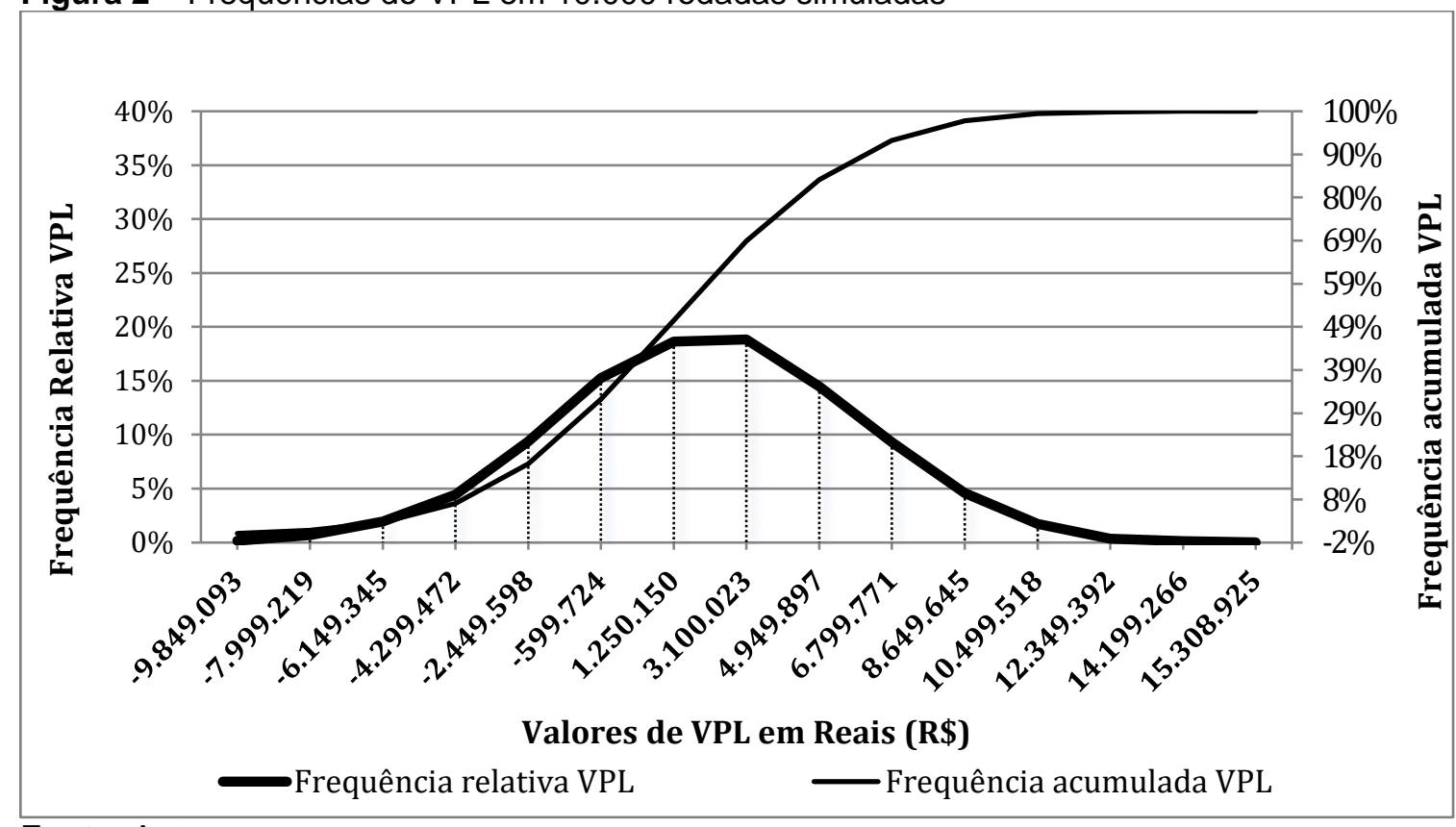

Fonte: Autores

De acordo com a distribuição acumulada da figura 2, pode se inferir a probabilidade de ocorrência para qualquer valor no intervalo de dados. Para encontrar uma probabilidade basta marcar o ponto de encontro entre a curva de frequência acumulada e o valor de VPL do eixo inverso a curva normal. Assim, observa-se na figura acima, que no ponto onde ocorre a interceptação da curva de frequência acumulada, com o eixo dos valores do VPL, encontra-se a probabilidade de ocorrência de valores menores ou iguais a zero.

A densidade de probabilidade, foi calculada pela equação 2.4 , da seção 3.2 (Modelo Analítico). A resposta a esta avaliação de risco, mostra que as possibilidades de valores menores do que zero para o VPL simulado, atingiram 39,7238\%, o que denota alto risco para o empreendimento, pois a possibilidade de que o índice de saída do modelo que avalia a saúde econômica do sistema seja negativo, aproximase de $40 \%$ entre todas as possibilidades consideradas dentro do modelo simulado. Ou seja, a combinação entre os valores de preços simulados geram aproximadamente 4.000 valores negativos de VPL, em um universo de 10.000 rodadas de simulações.

Respondendo a questão mais fundamental do trabalho: "Avaliar a taxa de risco financeiro que o investimento enfrentaria", verificaram-se fortes possibilidades de que os produtores assentados não suportariam tal risco no ambiente de negócios, baseando-se nas premissas de operação elaboradas para o sistema e com o método 
empregado. Esta afirmação fundamenta-se na análise simulada de risco de resultados negativos no VPL, avaliado em $40 \%$.

Cardozo et al. (2008), afirmam, para agricultores familiares, as alternativas acompanhadas de incertezas geralmente tem maior probabilidade de fracassar. No entanto, alternativas que colocam os produtores em melhores posições dentro de atividades onde estes já possuem experiência, conhecimento e vínculos comerciais parecem mais apropriadas para concentrar esforços.

Os resultados de risco, parecem mostrar-se como fortes obstáculos ao empreendimento no assentamento examinado, corroborando com a afirmação do autor supracitado, com relação as incertezas encontradas dentro do enquadramento da pesquisa.

Quanto as variáveis discutidas para se chegar até o resultado de risco encontrado, pode-se argumentar com relação a qualidade e a quantidade analisada. Neste sentido, o autor associa-se a Mendes e Padilha Junior (2007), que afirmam que os elementos de preços são adequados ao exame de investimentos, quando estes elementos gerarem grandes diferenças no VPL.

Portanto, os achados (avaliação da variação de preços) indicam uma colaboração para ampliar a base de informações para os decisores, pois mesmo num contexto de limitações diversas de um assentamento rural, quando se afirma de maneira categórica a respeito dos resultados analisados, no sentido de comunica-los por meio de conceitos quantitativos de fácil entendimento, estes tomadores de decisão podem agregar um elemento numérico que serve como um forte parâmetro, no que se refere ao requisito de resultado econômico do empreendimento.

Esta afirmação vai de encontro aos autores; Belfiore e Fávero (2012), quando estes preconizam que a tomada de decisão se identifica diretamente com os objetivos da organização, de modo a minimizar as incertezas e os riscos inerentes ao processo, e ainda escolher a decisão mais eficaz. Para isso, a qualidade das informações é fundamental. A experiência prévia, a observação empírica e o consenso geral podem gerar boas decisões, mas o uso de ferramentas que forneçam resultados em forma de informações de qualidade para o processo de decisão, podem embasar fortemente este processo e suportar decisões com maior grau de certeza.

Guardadas as formulações diferenciadas da racionalidade administrativa das pequenas propriedades familiares rurais, sob o ângulo da gestão e das relações com 
os meios de produção e de relacionamento com mercados e com seu local de moradia e trabalho, Lima et al. (1995), Payés e Silveira (1997), Navarro (2010) e Abramovay (2012), admitem frações da racionalidade administrativa no produtor familiar rural, voltadas para cenários capitalistas.

Batalha et al. (2005) afirmaram que o paradigma da agricultura familiar encontrava-se entre as suas características sujeitas a um contexto local e ao mesmo tempo inseridas sob uma competitividade de mercados bem mais amplos. Desta maneira os autores defendem a inserção de ferramentas gerenciais adequadas aos agricultores familiares respeitando as lógicas de mercado e o contexto destes produtores.

Não obstante, outra particularidade ou característica importante na tomada de decisão para agricultores familiares, citada por Cardozo et al. (2008) é o ingresso contínuo de valores. Ou seja, em uma situação de tomada de decisão onde os ingressos de valores mensais podem ter maior relevância sobre o resultado econômico, justifica-se mesmo que dentro da análise tradicional algum exame desta natureza. Cardozo et al. (2008), afirmam que o ingresso de valores no caso dos agricultores familiares, tem maior impacto em decisões administrativas de atividades produtivas, em comparação com a viabilidade econômica do capital em um empreendimento. Portanto, a renda mensal deve ser analisada.

Os autores, Meyer e Gomes (2016) também encontraram no elemento Renda Contínua, um item relevante aos assentados conforme apresentado no mapa cognitivo da pesquisa relatada pelos autores.

A expectativa de renda dos assentados, foi verificada através do cruzamento dos dados das entrevistas com líderes dos produtores, com os resultados do caixa projetado. Na opinião dos assentados (informação verbal) ${ }^{1}$ de Rosário do Sul, a remuneração mensal considerada satisfatória para uma atividade econômica desenvolvida pelo grupo seria 1,38 salários mínimos, para cada família, em média, ao mês. O resultado das projeções, demonstram que os valores médios de ingressos monetários, seriam suficientes para suprir no máximo 26 famílias.

\footnotetext{
1 Informação pessoal fornecida por Rogério Ustra, Presidente da Associação dos Produtores da Divisa, em conversa informal no assentamento Comunidade Divisa, em Rosário do Sul, novembro de 2013.

Revista Produção Online. Florianópolis, SC, v. 19, n. 4, p. 1120-1145, 2019
} 
Tabela 5 - Resultado médio mensal projetado/Família $(\mathrm{R} \$)$

\begin{tabular}{lll}
\hline a Salário Mínimo (R\$) & $\mathrm{R} \$ 724,0$ \\
b Fluxo de Caixa Médio Anual & $\mathrm{R} \$ 317.042,1$ \\
c Sobra de Caixa Médio Mensal ("b"/12) & $\mathrm{R} \$ 26.420,2$ \\
d Valor/Família/Mês ("c"/60) & $\mathrm{R} \$ 440,3$ \\
e Salário/Família/Mês ("d"/"a") & 0,61 \\
\hline
\end{tabular}

Fonte: Autores

Os resultados da tabela (Tabela 5) acima, mostram que o processo de simulação, envolvendo atividades projetadas nas suas fases iniciais, também podem auxiliar a base de decisões onde a renda contínua pode ter um "peso" maior em decisões gerenciais.

O desenvolvimento de cenários de atividades, partindo de projetos técnicoeconômicos, submetidos a simulações de Monte Carlo, denota uma abordagem sistêmica, como ferramenta de apoio a pequenos produtores em trabalho associativo.

Batalha et al. (2005), comentam a deficiência em análises que apoiem critérios de definição de produto e de processo, apresentados em longo prazo, juntamente com planos logísticos entre outras deficiências comuns dos esforços das pesquisas em ampliar estudos sobre tecnologias de gestão para agricultura familiar.

Andoseh et al. (2014) afirmaram que a Agencia para o Desenvolvimento Internacional dos Estados Unidos (USAID), com base em estudos de viabilidade econômica para cultivo de variedades melhoradas para culturas de importância na segurança alimentar de Uganda, recomenda o aprofundamento das bases para projetos públicos de investimento na agricultura, por meio do estudo de opções reais flexibilizadas que permitam visualizar além do cenário tradicional de viabilidade em termos de VPL. Os autores justificam dizendo que o método tradicional de análise (VPL) não é capaz de expor incertezas de maneira eficiente e que para analisar projetos agrícolas são necessários múltiplos estágios de testes para formar uma análise robusta de um projeto de investimentos.

A criação de um modelo eficiente em todos os aspectos da produção e comercialização, ultrapassa as pretensões do trabalho aqui apresentado, no entanto, a idéia de utilizar modelos que ampliem as possibilidades de visualização de cenários possíveis, pode ser considerada uma alternativa importante para compor bases de decisões em trabalhos associativos para produtores rurais. 
Assim, a utilização do modelo simulado, estruturado no trabalho, com a finalidade de examinar um negócio dentro do assentamento, forneceu elementos que permitiram que uma decisão totalmente nova, com poucos parâmetros para uma escolha, ganhasse maior qualidade, através dos elementos de preços simulados. Fato que pode ajudar a nortear o caminho de escolha para atividades econômicas para estes assentados.

Ainda sobre as potencialidades do uso de simulações de Monte Carlo em análises de risco para projetos em atividades para assentamentos rurais, Gonzales (1997) comenta que entre as vantagens dessas simulações, a flexibilidade de aplicação de regras e de incorporação de novos elementos se destaca para esta prática. O autor afirma que uso de simulações em experimentos de interações complexas parecem avaliar de maneira satisfatória, algumas variações no sistema, e assim mostrar informações das quais se tem pouca idéia das suas dimensões até serem vistas num quadro de probabilidades calculadas. Os modelos de simulação podem servir como teste para delinear novas políticas e regras para tomar decisões de investimento coletivo. Mesmo reconhecendo que uma abordagem analítica das partes de um sistema de produção familiar não é capaz de explicar totalmente a racionalidade administrativa destes grupos de produtores.

Para Gonzales (1997), as desvantagens de modelos simulados repousam nos conhecimentos múltiplos necessários para sua construção, e a na fase de validação de um modelo que pode representar grandes dificuldades.

Observa-se no caso da pesquisa, além de uma interação de múltiplos conhecimentos, a dependência, ou a ancoragem das simulações em projetos de investimento que merecem cuidados especiais inclusive com as questões das limitações naturais da região analisada.

\section{CONSIDERAÇÕES FINAIS}

A taxa de risco financeiro sob o efeito das simulações de resultado no investimento, enfrentaria com base nas premissas elaboradas, uma taxa de risco, incompatível com a condição de aceitação de um projeto por assentados rurais.

A proposição de atividades no assentamento, através da análise econômica tradicional de negócios, apresentou argumentação capaz de fornecer elementos `a Revista Produção Online. Florianópolis, SC, v. 19, n. 4, p. 1120-1145, 2019 
decisão de empreender, no entanto, essa análise mostrou-se insuficiente quando utilizada como única fonte de informações no apoio a decisões desta natureza. A simulação do tipo Monte Carlo estendeu o entendimento de possibilidades, complementando a análise econômica tradicional, no caminho da elucidação de riscos de preços combinados nas duas atividades.

Ampliar as variáveis analisadas, validar o sistema e acrescentar enfoques sociais e culturais próprios do grupo, assim como possíveis externalidades, poderiam tornar mais robusto o modelo analisado

\section{REFERÊNCIAS}

ABRAMOVAY, R. Paradigmas do capitalismo agrário em questão. São Paulo: Edusp, 2012.

ANDOSEH, S.; BAHN, R.; GU, J. The case for a real options approach to ex-ante costbenefit analyses of agricultural research projects. Food Policy, v. 44, p. 218-226, 2014. https://doi.org/10.1016/j.foodpol.2013.09.016

ANDRADE, E. L. Introdução à pesquisa operacional: métodos e modelos para análise de decisão. 2. ed. Rio de Janeiro: LTC S.A., 2000.

BANA E COSTA, C. A. Três convicções fundamentais na prática do apoio à decisão. Pesquisa Operacional, v. 13, n. 1, p. 9-20, 1993.

BANCO Central do Brasil. Histórico das taxas de juros fixadas pelo Copom e evolução da taxa Selic. 2014. Disponível em: https://www.bcb.gov.br/?COPOMJUROS. Acesso em: 24 mar. 2014.

BATALHA, M. O.; BUANAIN, A. M.; H. M. SOUZA, F. Tecnologia de gestão e agricultura familiar. gestão integrada da agricultura familiar. São Carlos: EduFScar 2005.

BELFIORE, P.; FÁVERO, L. P. Pesquisa operacional para cursos de administração, contabilidade e economia. Rio de Janeiro: Elsevier, 2012

BERGAMASCO, S. M. P. P. A. Realidade dos Assentamentos Rurais por Detrás dos Números. Revista Estudos Avançados, São Paulo, v. 11, p. 37-49, 1997. https://doi.org/10.1590/S0103-40141997000300003

BERTOLO, L. A. Risco, Orçamento de Capital e Diversificação. Finanças no Excel 2007, 2008. Disponível em: www.bertolo.pro.br/FinEst/Financas/Livro/CAPITULO\%2011.pdf Acesso em: 11 de ago. 2014.

BRUNI, A. L.; FAMÁ, R.; SIQUEIRA, J. O. Análise do risco na avaliação de projetos de investimento: uma aplicação do método de Monte Carlo. Caderno de Pesquisas em Administração, v. 01, n. 06, p. 62-75, 1998.

BUSSAB, W. O.; MORETTIN, P. A. Estatística básica. 6. Ed. São Paulo: Saraiva, 2010. 
CARDOZO, O. et al. Producción intensiva de carne vacuna en predios de area reducida. INIA. Montevideo. v. 175, p. 100, 2008.

CHWIF, L.; MEDINA, A. C. Modelagem e simulação de eventos discretos: teoria e aplicações. 3. ed. São Paulo: Bravarte, 2010.

CURY, M. V. Q. Estatística: curso de educação continuada. Rio de Janeiro: Fundação Getúlio Vargas, 2004.

CHWIF, L.; MEDINA, A. C. Modelagem e simulação de eventos discretos: teoria e aplicações. 3. ed. São Paulo: Bravarte, 2010.

CHUNG, C. A. Simulation modeling handbook: a practical approach. Florida: CRC, 2004. https://doi.org/10.1201/9780203496466

FÁVERO, L. P.; BELFIORE, P. Pesquisa operacional para cursos de administração, contabilidade e economia. Rio de Janeiro: Elsevier, 2012.

FERNANDES, C. A. B. A. Gerenciamento de riscos em projetos: como usar o microsoft excel para realizar a simulação Monte Carlo. Curitiba: Centro Federal de Educação Tecnológica do Paraná 2005.

FRANÇA, F. M. C.; HOLANDA JÚNIOR, E. V.; SOUSA NETO, J. M. Análise da viabilidade financeira e econômica do modelo de exploração de ovinos e caprinos no Ceará por meio do sistema agrossilvipastoril. Brasília: PRODETAB, EMBRAPA e IICA. v. 42, n. 11, p. 287-308, 2011.

GÁL, T. M.; NAGY, L.; DÁVID, L. R. N.; VASA, L. S.; BALOGH, P. T. Technology Planning System as a Decision Support Tool for Dairy Farms in Hungary. Acta Polytechnica Hungarica, v.10, 2013. https://doi.org/10.12700/APH.10.08.2013.8.15

GAVIRA, M. O. Simulação computacional como uma ferramenta de aquisição de conhecimento. 2003. 150f. Dissertação (Mestrado em Engenharia de Produção) - Escola de Engenharia de São Carlos, Universidade de São Paulo, São Carlos, 2003.

GONZALES, C. A. Simulacion de sistemas: aplicaciones em produccion animal. Santiago, Chile: Pontifícia Universidad Católica de Chile, 1997.

LAPPONI, J. C. Projetos de investimento: construção e avaliação do fluxo de caixa: modelos em excel. São Paulo: Lapponi Treinamento, 2000.

LEITE, S. Orçamentos familiares e estratégias socioeconômicas em assentamentos rurais. Revista Estudos Sociedade e Agricultura. Rio de Janeiro, v. 21, p. 118-151, 2003.

LIMA, A. P. B. et al. Administração da unidade de produção familiar: modalidades de trabalho com agricultores. 3.ed. ljuí,1995.

LYRA, G. B. et al. Viabilidade econômica e risco do cultivo do mamão em função da lâmina de irrigação e doses de sulfato de amônio. Acta Scientiarum Agronomy, v. 32, n. 3, p. 547554, 2010. https://doi.org/10.4025/actasciagron.v32i3.2451

MENDES, J. T. G.; PADILHA JUNIOR, J. B. Agronegócio: uma abordagem econômica. Pearson Prentice Hall, 2007.

Revista Produção Online. Florianópolis, SC, v. 19, n. 4, p. 1120-1145, 2019 
MEYER, A. A.; GOMES, M. C. Apoio a decisão de negócio em assentamento rural utilizando uma metodologia multicriterial. Espacios. Caracas, v. 37, 2016.

MONTE, P. A.; PEREIRA, A. E. S. Um Estudo Regional dos Determinantes da Geração de Renda e Construção da Cidadania nos Projetos de Assentamentos. Revista de Economia e Sociologia Rural, v. 47, p. 1023-1040, 2009. https://doi.org/10.1590/S0103$\underline{20032009000400010}$

MOREIRA, R. C. et al. Viabilidade econômica da agroindústria familiar rural de Frutas na Zona da Mata Mineira. Revista de Economia e Agronegócio. v. 5, p. 187-206, 2007.

NARDELLI, P. M.; MACEDO, M. A. S. Análise de um Projeto Agroindustrial Utilizando a Teoria de Opções Reais: a opção de adiamento. Economia e Sociologia Rural. São Paulo, v. 4, p. 941-966, 2012. https://doi.org/10.1590/S0103-20032011000400006

NAVARRO, Z. A agricultura familiar no Brasil: entre a política e as transformações da vida econômica. In: GASQUES, J. G.; VIEIRA FILHO, J. E. R.; NAVARRO, Z. A agricultura brasileira: desempenho, desafios e perspectivas. Brasília: IPEA, 2010.

NORDER, L. A. C. Assentamentos rurais: casa, comida e trabalho.151 Dissertação (Mestrado) - Instituto de Filosofia e Ciências Humanas, Unicamp, Campinas, 1997.

NORONHA, J. F. Projetos agropecuários: administração financeira, orçamento e viabilidade econômica. 2. Ed. São Paulo: Atlas, 1987.

PAYÉS, A. M.; SILVEIRA, M. A. A Racionalidade econômica do empresário familiar. CNPMA, C. N. D. P. D. M. E. A. D. I. A. Jaguariúna,SP: Embrapa, 1997.

PICCIN, M. B. Assentamentos Rurais e Geração de Renda: Posição Social Restringida, Recursos Socioculturais e Mercados. Economia e Sociedade. Campinas, v. 21, p. 115-141, 2012. https://doi.org/10.1590/S0104-06182012000100005

PONCIANO, N. J. et al. Análise de viabilidade econômica e de risco da fruticultura na Região Norte Fluminense. Revista de Economia e Sociologia Rural. v. 42, p. 615-635, 2004. https://doi.org/10.1590/S0103-20032004000400005

PRADO, D. Teoria das filas e da simulação. 4. ed. Belo Horizonte: Nova Lima, 2009.

SILVA, W. F. Contribuição da simulação de Monte Carlo na projeção de cenários para gestão de custos na área de laticínios. 2004. 137 Dissertação (Mestrado) - Engenharia de Produção, Universidade Federal de Itajubá, Itajubá.

SIMON, H. A. Administrative behavior: a study of decision-making processes in administrative organizations. 4.ed. New York: 1997.

SOLANO, C. et al. Who makes farming decisions? a study of Costa Rica dairy farmers. Agricultural Systems, v. 67, p. 181-199, 2001. https://doi.org/10.1016/S0308-521X(00)00053$\underline{6}$

STATPLUS®: Mac LE. Analyst Soft Inc: programa de análise estatística. 2009. Disponível em: http://www.analystSoft.com/br/. 
STEVENSON, W. J. Estatística aplicada à administração. São Paulo: Harper \& Row do Brasil, 1981

TAVARES, B. S. et al. Análise de risco e otimização de recursos hídricos e retorno financeiro em nível de fazenda. Revista Brasileira de Engenharia Agrícola e Ambiental. Campina Grande. v. 15, p. 338-346, 2011. https://doi.org/10.1590/S1415-43662011000400002

TRIGEORGIS, L.; MASON, S. P. Valuing managerial flexibility. Midland Corporate Finance Journal, v. 5, n. 1, p14-21, 1987.

ZDANOWICZ, J. E. Fluxo de caixa: uma decisão de planejamento e fluxo financeiros. 5. ed. Porto Alegre: Sagra Luzzatto, 1992.

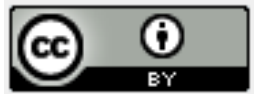

Artigo recebido em: 03/10/2017 e aceito para publicação em: 12/01/2019

DOI: http://dx.doi.org/10.14488/1676-1901.v19i4.3025 